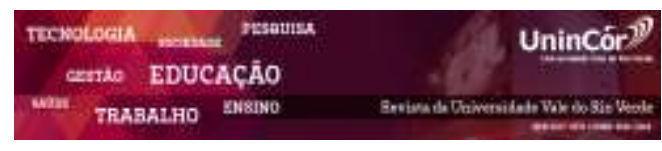

Revista da Universidade Vale do Rio Verde ISSN: 1517-0276 / EISSN: 2236-5362 Vol. 16 | n. 1 | Ano 2018

\section{MUDANÇA NO MODELO ASSISTENCIAL NA ATENÇÃ̃O PRIMÁRIA: PERSPECTIVA DOS USUÁRIOS}

\section{RESUMO}

Em Portugal, a atenção primária à saúde tem sido alvo de várias mudanças, nomeadamente com o desenvolvimento de Unidades de Saúde Familiar (USF) que foram gradualmente substituindo as Unidades de Cuidados de Saúde Personalizados. No Brasil segundo a literatura a estratégia para a expansão da atenção primária se realizou por meio da Estratégia de Saúde Familiar (ESF) que foi substituindo as Unidades Básicas de Saúde (UBS). A presente pesquisa teve como objetivo, por um lado avaliar as alterações no modelo assistencial segundo a percepção dos usuários da Região de Lisboa e, por outro, estabelecer uma comparação com o Brasil por meio dos resultados de um estudo publicado na Revista da Universidade Vale do Rio Verde, Três Corações. Trata-se de uma pesquisa qualitativa, com coleta de dados realizada por aplicação de 20 entrevistas semiestruturadas a usuários frequentadores que foram analisadas com recurso à análise de conteúdo. Como principais conclusões se verificou uma satisfação dos usuários com o novo modelo, identificaram-se muitas semelhanças com os resultados obtidos no Brasil, nomeadamente na melhoria da acessibilidade, atendimento e na capacidade de resposta e algumas diferenças que poderão constituir pontos de melhoria para ambas as experiências.

Palavras-chave: Atenção Primária à Saúde; Unidade de Saúde Familiar; Estratégia de Saúde da Família.

\section{CHANGE IN THE ASSISTANCE MODEL IN PRIMARY CARE: USERS PERSPECTIVE}

\begin{abstract}
In Portugal, primary health care has undergone several changes, in particular with the development of Family Health Units (USF) that have been gradually replacing the Personalized Health Care Units. In Brazil, according to the literature, the strategy for the expansion of primary health care was carried out through the Family Health Strategy (ESF), which replaced the Basic Health Units (UBS). The objective of this study was to evaluate the changes in the care model according to the perception of the users of the Lisbon region and, on the other hand, to compare with Brazil through the results of a study published in the Revista da Universidade Vale do Rio Verde, Three Hearts. It is a qualitative research, with data collection performed through the application of 20 semi-structured interviews to users who were analyzed using content analysis. The main conclusions were that users were satisfied with the new model, many similarities were identified with the results obtained in Brazil, namely in the improvement of accessibility, service and response capacity and some differences that could be points of improvement for both experiences.
\end{abstract}

Keywords: Primary Health Care; Family Health Unit; Family Health Strategy. 


\section{INTRODUÇÃO}

A atenção primária à saúde é considerada pelo Ministério da Saúde como a principal porta de entrada no Serviço Nacional de Saúde (NUNES, 2016) e tem evoluído ao longo das últimas décadas, buscando sempre um modelo adequado que permita uma melhor assistência.

É neste nível de atenção que são realizadas as atividades de promoção da saúde, prevenção da doença por meio de uma abordagem centrada na pessoa, família e comunidade (BISCAIA e HELENO, 2017).

As consultas em atenção primária são realizadas por médicos e por enfermeiros num conjunto vasto de serviços, nomeadamente: cuidados médicos gerais para a população adulta; cuidados pré-natais; cuidados infantis; saúde da mulher; planejamento familiar; primeiros socorros; visitas ao domicilio; rastreamento do câncer e vacinação. No entanto, existem algumas unidades que contam com médicos especialistas nas áreas de saúde mental, psiquiatria, dermatologia, pediatria, ginecologia e obstetrícia e cirurgia (BISCAIA e HELENO, 2017; SIMÕES et al., 2017).

De financiamento exclusivamente público, a atenção primária em Portugal, desde 2007, está organizada em 55 Agrupamentos de Centros de Saúde (ACES) que são compostos por várias unidades (Centros e postos de saúde). $\mathrm{O}$ número de centros e postos de saúde foi crescendo ao longo dos anos, passando de 329 em 1985 para 357 desde 2012 (INE, 2018). O objetivo futuro da prestação de cuidados de saúde primários em Portugal passa pela completa cobertura de Unidades de Saúde Familiar (USF) que são unidades funcionais existentes nos ACES que prestam cuidados mais personalizados aos seus utentes numa perspectiva de proximidade e de acompanhamento do estado de saúde (BISCAIA e HELENO, 2017).

As USF são as unidades elementares de prestação de cuidados de saúde, individuais e familiares, baseadas em equipas multidisciplinares que integram: médicos, enfermeiros e pessoal administrativo (DECRETO-LEI n. ${ }^{\circ}$ 298/2007, de 22 de agosto) e que podem contar ainda com a participação de outros profissionais como médico dentista, higienista oral, psicólogo, assistente social.

As USF têm como missão a assistência personalizada à população inscrita em uma determinada área geográfica, garantindo o acesso, a globalidade, a qualidade e a continuidade da assistência.

O funcionamento das USF é baseado em um plano de ação que representa um compromisso assistencial formalizado anualmente e que traduz o programa de atuação e o compromisso das USF com os respetivos objetivos assistências, considerando os objetivos de acesso, eficiência e qualidade e respetivas métricas de avaliação. Assim, nesse plano deve indicar: o portfólio básico de serviços; os horários de funcionamento; o sistema de marcação, atendimento e orientação dos usuários; a definição do sistema de renovação de 
prescrições; a articulação com outras unidades e a carteira de serviços adicionais.

O processo de trabalho é voltado para o cidadão e obedece aos seguintes princípios:

1. Conciliação - garantir a assistência personalizada;

2. Cooperação - exige que todos os elementos da equipa se esforcem para a realização dos objetivos garantindo o acesso e a continuidade da assistência;

3. Solidariedade - cada elemento da equipe deve assumir a sua responsabilidade para garantir obrigações dos restantes elementos;

4. Autonomia - baseada na auto-organização funcional e técnica de cada equipe, respeitando o plano de ação;

5. Articulação - estabelecimento de uma conexão entre a atividade desenvolvida pelas USF e as outras unidades;

6. Avaliação - é realizada de forma permanente tendo por base os objetivos préestabelecidos no plano de ação;

7. Gestão participativa - a gestão deve ser adoptada por todos os profissionais da equipe com o objetivo de melhorar o desempenho e promover a satisfação profissional. (Artigo $5^{\circ}$ DECRETO-LEI n. ${ }^{\circ}$ 298/2007, de 22 de agosto).

Este novo modelo surgiu no ano 2007 e desde então têm vindo a crescer (atualmente segundo Portal SNS existem 504 USF), substituindo as tradicionais Unidades de Cuidados de Saúde Personalizados (UCSP), também conhecidos como Centros de Saúde. Por sua vez, no Brasil como resultado da evolução do modelo assistencial na atenção primária à saúde, surgiu em 1994 a Estratégia de Saúde Familiar
(ESF) (BRASIL, 1998), que tem vindo a substituir nos vários municípios as tradicionais Unidades Básicas de Saúde (UBS) ou Centros de Saúde (MARTINS, et al. 2016; ONOCKOCAMPOS, 2012). Segundo Campos et al (2014), o acesso nas USB é dificultado por: problemas de organização; uso do acolhimento como barreira ao acesso; falta de recursos humanos; dificuldades em dar seguimento ao tratamento.

De forma identica ao caso das USF portuguesas, as ESF no Brasil integram atividades de promoção da saúde, prevenção da doença e assistência curativa (BRASIL, 1998) e são constituídas por equipes multiprofissionais que integram: médico, profissionais de enfermagem (enfermeiros e técnicos), agente comunitário e em alguns casos profissionais de saúde bucal. (CASTRO et al, 2012).

Tanto em Portugal, como no Brasil, a substituição das unidades tem sido gradual pelo fato de existiram muitas dificuldades na sua realização e alguns desafios nomeadamente os financeiros no caso português.

A organização do atual modelo de atenção primária foi bem classificada num recente estudo da OCDE (2015) pelo acesso, baixo custo e pela elevada qualidade da informação disponível com vários indicadores, o que não se verifica na maioria dos países da OCDE. O mesmo estudo destacou muito positivamente a reforma iniciada na atenção primária, nomeadamente a criação de Unidades de Saúde familiar que para os autores do estudo promoveu um maior acesso, com maior qualidade, eficiência e satisfação dos utentes.

No entanto, mesmo considerando importante a avaliação internacional, segundo 
autores como Assis e Jesus (2012), Ramos e Lima (2003), Campos et al. (2014) e Milan e Trez (2005), é ainda mais pertinente a opinião dos usuários e é com base nela que se pode melhorar a assistência na atenção primária e os resultados dos indicadores operacionais de desempenho e pode estar segundo Starfield (2002) relacionada com a qualidade.

O presente estudo teve como objetivo avaliar a satisfação dos usuários relativamente à assistência prestada pela equipe das USF na Região de Lisboa e Vale do Tejo e ainda foi estabelecido como objetivo comparar os resultados com os obtidos no Brasil por meio de um estudo publicado na Revista da Universidade Vale do Rio Verde, Três Corações que focou a avaliação da satisfação dos usuários de uma ESF implementada em substituição de uma UBS no município de Montes Claros, Minas Gerais.

\section{MÉTODOS}

A presente pesquisa trata-se de uma pesquisa qualitativa de caráter analítico realizada por meio da aplicação de entrevistas semiestruturadas desenvolvida entre 02 de janeiro a 13 de março de 2018.

Como pesquisa qualitativa, o estudo pretendeu explorar e descrever a percepção dos usuários em seu ambiente natural de forma a melhor entender o foco do fenómeno em estudo (ALMEIDA; FREIRE, 2003; BAPTISTA; CAMPOS, 2007). No presente caso, avaliando a realidade vivida e sentida pelos usuários da atenção primária à saúde que assistiram à substituição do modelo de assistência.
A coleta de dados ocorreu junto de 20 usuários (10 homens e 10 mulheres) com idade compreendida entre os 40 e os 70 anos, cadastrados em 4 unidades de saúde familiar, que possuem tempo de moradia superior a 10 anos e que utilizaram os serviços no mesmo local nas duas modalidades (USCSP e USF).

A pesquisa respeitou todas as normas éticas existentes em Portugal, foi submetido ao Comitê de Ética em Pesquisa da Autoridade Local de Saúde e todos os entrevistados assinaram um termo de consentimento livre e esclarecido de forma a informar os entrevistados do tema, objetivos e direitos resguardados e a permitir a análise e publicação dos dados.

A aplicação das entrevistas à população alvo foi precedida de um teste. As entrevistas com o objetivo de garantir uma melhor análise das falas foram gravadas e transcritas e ocorreram no domicílio dos entrevistados. A duração aproximada de cada entrevista foi de 20 minutos, não existindo recursa por parte de nenhum participante convidado a dar o seu depoimento. De forma a garantir o anonimato, os entrevistados são representados pela letra e seguida de um número (código de identificação). De forma a identificar as unidades de significação mais relevantes relativamente à percepção dos usuários em relação aos resultados da assistência na atenção primária com o novo modelo de organização, a análise da informação coletada foi submetida à análise de conteúdo. Segundo Bardim (2006) e Minayo (2013), este método assume especial relevância na análise de dados recolhidos por meio de entrevistas, pelo fato de incorrer num processo de codificação, por meio de um processo de interpretação das 
palavras que permite uma inclusão/agrupamento das falas relevantes em categorias. Esse processo decorreu em três etapas:

1.Etapa de pré-análise (várias leituras das falas para identificar pontos comuns e divergentes);

2.Definição de categorias e subcategorias;
3.Tratamento dos dados e discussão confrontando os achados com o caso das ESF obtidos no estudo de Martins, et al. (2016) publicado na Revista da Universidade Vale do Rio Verde, Três Corações.

\section{RESULTADOS E DISCUSSÃO}

Após as várias leituras e identificação dos vários pontos referidos pelos usuários se procedeu à categorização, surgindo 3 categorias e 13 subcategorias conforme se demonstra na Tabela 1:

Tabela 1 - Categorias e subcategorias sobre a mudança do modelo assistencial na perspectiva dos usuários em quatro USF da região de Lisboa.

\begin{tabular}{c|c|c|c}
\hline Categorias & Acessibilidade & Atendimento (Relação) & Resolubilidade \\
\hline & Disponibilidade; & Recebimento; & Novas respostas; \\
& Tempos de espera; & Escuta ativa; & Realização de exames; \\
Subcategorias & Encaminhamento para & Ações de educação; & Articulação e Continuidade; \\
& especialistas; & Contato & Acesso à informaço da atividade; \\
& Agendamento clínica. & &
\end{tabular}

Fonte: Elaborado pelo autor

\section{Apresentação das categorias e subcategorias}

\section{Categoria I - Acessibilidade}

A melhoria da acessibilidade na atenção primária no modelo de USF foi destacada por todos os entrevistados nas suas falas, referindo alguns pontos fortes como são exemplo:

\section{a) Disponibilidade (marcação de consulta)}

A uma maior disponibilidade é relatada por $90 \%$ dos entrevistados, que fizeram referência ao alargamento do horário, à maior oferta para marcação de consultas e ao menor tempo de espera para agendamento.

E1: "Antes esperava meses para poder ter uma consulta. Agora o horário alargou e as consultas começam logo pelas 08h e vão até às $20 h$. Consigo marcação em menos de uma semana".
E16: "Esperava e desesperava para ter consulta, mas hoje é muito mais fácil".

Segundo a literatura, a disponibilidade para receber os pacientes é fundamental na atenção primária à saúde, pois é a principal porta de entrada no sistema de saúde português (NUNES, 2016) e também no Brasil (STARFIELD, 2002). Por meio da promoção da acessibilidade é possível dar uma melhor resposta às necessidades da população (ACSS, 2016; SIMÕES, 2017).

\section{b) Tempo de espera}

$85 \%$ dos usuários relatam um menor tempo de espera para atendimento no dia da consulta, isso reflete uma maior comodidade para os usuários.

E5: "No modelo anterior chegava às $8 h$ para marcar a vez (ordem de chegada) mas como o 
médico só entrava às $10 \mathrm{~h}$ era atendida perto das 12h. Agora o médico chega na hora certa e tenho hora marcada"

E16: "A consulta agora tem no máximo meia hora de atraso ".

\section{c) Agendamento}

Um outro aspeto que segundo Mendes (2011) contribui para a maior comodidade dos usuários no contato com os serviços de saúde é a maior facilidade no agendamento. Com o novo modelo surgiram também mais formas de agendamento que são relatadas pelos entrevistados:

E2: "Pego no telefone e faço a marcação, não sendo necessário ir para o centro de saúde presencialmente de madrugada como acontecia" E7: "Vou à internet e no meu perfil pessoal de saúde do portal do cidadão tenho acesso à marcação de consultas, recebendo um SMS e email confirmando o agendamento"

\section{d) Encaminhamento para especialistas}

Os usuários nas suas falas reconhecem uma maior facilidade no encaminhamento para as consultas de especialidade, muitas delas realizadas no hospital de proximidade, referindo que são marcadas por meio do novo sistema informático implementado com o novo modelo da atenção primária.

E14: "O médico da atenção primária faz a marcação online e sou atendida rapidamente".

E15: "Não tenho razão de queixa os exames especializados são pedidos pelo médico no momento e não há papel de marcação, é tudo realizado online e muito rápido".

Segundo Fernandes (2015), a rapidez no acesso muitas das vezes se deve à combinação pública privada, pois sempre que não há capacidade de resposta interna ou qualquer limitação de acesso nomeadamente a exames laboratoriais e radiológicos é solicitado apoio ao setor privado local que tem acordos estabelecidos com as unidades de atenção primária.

A atenção primária em Portugal tem um impacto positivo na melhoria dos indicadores de saúde, nomeadamente por intervir não apenas na vertente curativa mas também na preventiva e nas ações de educação para a saúde, reduzindo emergências e internações (SIMÕES et al, 2017). O mesmo, segundo Mendes (2011), ocorre no Brasil, no entanto, contrariamente ao caso brasileiro, em Portugal existe uma fraca capacidade de resposta especializada na atenção primária existindo muitos encaminhamentos para a atenção secundária.

\section{Categoria II - Acolhimento (Relação Usuário- Profissional)}

A categoria do acolhimento, medido em termos da relação entre usuários e profissionais, é destacada nas falas como um dos aspetos que melhorou. Todos os usuários relatam que a equipa os recebe melhor, com maior capacidade e tempo para os ouvir (escuta ativa) e ainda promove ações de educação em saúde. Ou seja, com o modelo de USF saiu reforçada a confiança dos usuários na equipe de atenção primária.

\section{a) Recebimento dos usuários;}

Os usuários relataram que são recebidos de forma diferente pela equipe de saúde, destacando que no novo modelo têm um recebimento cordial, são tratados pelo nome e que antes da consulta médica ou de enfermagem os profissionais consultam o processo e não 
fazem perguntas repetitivas sobre o historial clínico.

E9: "Todos me conhecem e sabem o meu nome. Antes quando entreva na receção perguntavam como me chamava, depois ia ao enfermeiro e ele perguntava novamente o nome e pedia para contar os meus problemas e chegava ao médico e ele perguntava tudo de novo".

E11: "Fico muito satisfeito quando a equipe conhece o meu processo pois significa que se inteiraram dele e estão preocupados com o meu estado de saúde".

Segundo Fortuna et al. (2017) e Garuzi (2014), Klein e Gonçalves (2005), Lima et al., (2014) e Sousa et al., (2008), o acolhimento é um importante momento que faz toda a diferença na percepção do usuário relativamente à unidade de saúde, pois um atendimento personalizado e humanizado gera empatia que permite uma maior aproximação, facilita toda a relação, fortalece o respeito e a adesão terapêutica e reforça a confiança na equipe de saúde. Tudo isso melhora os níveis de qualidade assistencial.

\section{b) Escuta ativa}

$70 \%$ dos usuários, curiosamente todos os que têm mais de 60 anos, referem que os profissionais promovem uma escuta ativa ouvindo as suas queixas, problemas e melhorias.

E5: "A enfermeira ouve-me com atenção"

E10: "Esta equipe tem mais tempo para os mais idosos e ouvem com muita atenção todas as queixas deste velho"

Segundo a literatura a escuta ativa representa uma parte fundamental na relação entre pacientes e profissionais e permite a compreender a pessoa e trocar informações clinicamente importantes (CAR e
SHEIKH,2003; MESQUITA e CARVALHO, 2014).

\section{c) Ações de educação em saúde}

Todos os usuários referiram que os profissionais de saúde, particularmente os enfermeiros, têm sempre algo a ensinar para ajudar a melhorar a saúde ou prevenir a doença por meio de ações de educação.

E2: "Estamos sempre a aprender com esta equipe. A enfermeira tem sempre uma estratégia que permite mudar alguns hábitos errados"

E9: "Quando o médico prescreve um novo medicamento, a enfermeira ensina como tomar".

As ações de educação em saúde são importantes para alterar os comportamentos dos cidadãos e permitir que eles adotem medidas positivas de promoção da saúde e prevenção da doença (ASHCROFT, 2015; FEIJÃO et al., 2008; McMANUS, 2013). Por exemplo, um estudo que enfatizou a importância da educação em saúde na saúde pública foi o realizado por Sales (2008) quando abordou o tema da prevenção e controle da dengue no Ceará.

\section{d) Contato}

$60 \%$ dos usuários referiram que a forma de contato neste no novo modelo é muito profissional e realizado em sala fechada com a máxima privacidade e com o maior sigilo.

E5: "O centro de saúde agora tem melhores condições e espaços para reunir com a equipe".

E8: "No anterior modelo só o médico tinha gabinete e sempre com porta aberta. Agora toda a equipe tem uma sala".

\section{Categoria 3 - Resolubilidade}

$70 \%$ dos entrevistados consideraram que nas novas unidades existe maior capacidade de 
resposta. Neste sentido, foi atribuída a categoria de resolubilidade que é o termo utilizado pelo Ministério da Saúde português relativamente ao reforço da resposta na atenção primária. A percentagem de respostas é inferior, pois das quatro Unidades de Saúde Familiar, apenas em três foram implementados os novos serviços. No presente ano será concluído o processo.

\section{a) Novas respostas}

Os utentes destacam a existência de assistência em saúde oral (médico dentista) e ainda a possibilidade de ter consulta de nutrição e psicologia.

E11: "Gosto muito de falar com a senhora psicóloga. Ela consegue entender-me bem".

E13: "Neste centro de saúde há mais profissionais além dos médicos e enfermeiros".

\section{b) Realização de exames}

Os utentes destacam a existência de exames de cardiologia (ECG, holter, monitorização da pressão arterial), radiografia simples, análises clinicas e espirometrias.

E3: "Agora podemos realizar análises sem ter de ir a outro lado. Faço logo aqui"

E17: "Sou doente cardíaco e estou sendo monitorizado aqui no centro de saúde. Antes tinha de me deslocar ao hospital".

\section{c) Articulação e continuidade}

A articulação de cuidados de saúde é uma prioridade do Ministério da Saúde. Desde 2016, tem sido reforçada o encaminhamento e o seguimento dos pacientes desde a atenção primária para os cuidados hospitalares e póshospitalares (conhecidos como continuados em Portugal). Isso permite a melhoria da circulação dos pacientes no sistema e a redução de listas de espera (ACSS, 2016; SIMÕES, 2017). Starfield
(2002), retratando o caso brasileiro, refere que a continuidade dos cuidados permite conhecer melhor o paciente, reduzindo internações e custos associados. Os pacientes sentem isso e referem nas suas falas.

E4: "O médico encaminha o meu caso para o hospital e quando regresso ao centro de saúde o meu médico sabe o que aconteceu. É clara a ligação e articulação existente."

E11: "Desde que existe este novo modelo na atenção primária, sinto-me mais vigiado e nunca mais tive necessidade de ir ao serviço de emergências do hospital'.

\section{d) Acesso à informação clínica.}

O sucesso da articulação e continuidade dos cuidados é também muito devido aos sistemas de informação que foram desenvolvidos e que permitem ao médico da atenção primária numa perspectiva de cuidados personalizados conhecer o percurso do paciente no sistema de saúde podendo encaminhar e acessar a todas as informações.

\section{e) Programação da atividade}

O desenvolvimento dos sistemas de informação e a promoção da perspectiva de articulação e continuidade dos cuidados permite à equipe de saúde conhecer melhor os seus pacientes e agendar consultas e personalizar a resposta de acordo com as necessidades. Os pacientes nas suas falas sentem isso:

E14: "No antigo modelo ninguém me queria cá para realizar consultas, era mais uma entre muitos outros velhos, mas agora telefonam-me para cá vir"

E16: "Quando tenho uma consulta o meu médico marca logo a seguinte, não preciso de pedir ele agenda logo". 


\section{Comparação de resultados entre novo modelo}

\section{assistencial português e brasileiro}

Após a descrição das categorias e subcategorias registrada nas entrevistas realizadas aos usuários de Unidades de Saúde Familiar portuguesas, cabe analisar e comparar com os resultados obtidos no estudo realizado por Martins et al, (2016), que serviu de base à realização da presente pesquisa. A tabela 2 estabelece essa comparação relativamente às principais categorias.

Tabela 2 - Resultados comparados na experiência Brasileira e Portuguesa relativamente à mudança do modelo assistencial na perspectiva dos usuários em quatro USF da região de Lisboa.

\begin{tabular}{|c|c|c|}
\hline & Brasil & Portugal \\
\hline Acessibilidade & $\begin{array}{l}\text { Maior disponibilidade de ofertas de vagas } \\
\text { Maior comodidade (redução do tempo de } \\
\text { espera, alargamento do horário, } \\
\text { agendamento); } \\
\text { Maior capacidade de resposta da atenção } \\
\text { primária que tem especialistas como por } \\
\text { exemplo cirurgião dentista; } \\
\text { Dificuldade de marcação de consultas e } \\
\text { exames especializados. }\end{array}$ & $\begin{array}{l}\text { Maior disponibilidade; } \\
\text { Alargamento do horário de atendimento; } \\
\text { Menor tempo de espera para marcação; } \\
\text { Agendamento facilitado; } \\
\text { A atenção primária tem ainda poucos médicos } \\
\text { especializados pois estão muito centrados na } \\
\text { atenção secundária; } \\
\text { Facilidade de marcação de consultas e exames } \\
\text { especializados, realizada online e agendada no } \\
\text { momento da consulta. }\end{array}$ \\
\hline Acolhimento & $\begin{array}{l}\text { Fortalecida relação profissional-usuário; } \\
\text { Profissionais com atitude mais acolhedora; } \\
\text { Melhores atos de receber; } \\
\text { Maior capacidade para escutar as pessoas. }\end{array}$ & $\begin{array}{l}\text { Melhor recebimento dos pacientes; } \\
\text { Melhores condições das estruturas; } \\
\text { Profissionais ouvem os pacientes; } \\
\text { Praticadas ações de educação em saúde. }\end{array}$ \\
\hline Resolubilidade & $\begin{array}{l}\text { Novas respostas; } \\
\text { Implantação de novos serviços; } \\
\text { Assistência continuada ao paciente (visita } \\
\text { domiciliar, ações de promoção da saúde e } \\
\text { prevenção de doenças); } \\
\text { Incorporação do agente comunitário de saúde; } \\
\text { Maior capacidade técnica dos profissionais } \\
\text { (componente clínica e de comunicação); } \\
\text { Incorporação da abordagem familiar e } \\
\text { comunitária. }\end{array}$ & $\begin{array}{l}\text { Maior capacidade de resposta (assistência em } \\
\text { saúde oral (médico dentista), consulta de } \\
\text { nutrição e psicologia); } \\
\text { Exames de cardiologia (ECG, holter, } \\
\text { monitorização da pressão arterial), radiografia } \\
\text { simples, análises clinicas e espirometrias; } \\
\text { Articulação de cuidados de saúde; } \\
\text { Continuidade de cuidados; } \\
\text { Acesso à informação clínica } \\
\text { Planejamento da atividade. }\end{array}$ \\
\hline
\end{tabular}

Fonte: Elaborado pelo autor

Assim de acordo com a informação da tabela 2 foram encontrados muitos pontos comuns revelam uma melhoria na acessibilidade, acolhimento e resolubilidade em ambas as experiências. No entanto, existem pontos divergentes que poderão servir de base à melhoria tanto de um como de outro modelo. Assim:

a) $\mathrm{Na}$ experiência brasileira se verifica pontos fortes que poderão ser adotados por Portugal como são exemplo:
- Profissionais com atitude mais acolhedora;

- Promoção da visita domiciliar;

- Incorporação do agente comunitário;

- Aposta na melhoria da capacidade técnica e de comunicação verbal e não-verbal dos profissionais.

b) Como pontos fortes da experiência portuguesa que poderão ser adotados pelo Brasil, pode-se referir:

- Investimento em sistemas de informação e desenvolvimento de medidas de gestão que promovam a marcação de consultas e

- Existência de cirurgião dentista; 
exames especializados (partilha de recursos entre unidades);

- Implementação de exames especializados básicos nas unidades de atenção primária (ex: cardiologia, radiologia e espirometrias);

\section{CONCLUSÃO}

De acordo com a presente pesquisa verificou-se que a atenção primária à saúde em Portugal e no Brasil passaram por um recente processo de reforma que terminou com o desenvolvimento de USF no Serviço Nacional de Saúde Português e de Equipas de Saúde Familiar no Sistema Único de Saúde Brasileiro.

De acordo com a presente pesquisa, em Portugal, segundo os depoimentos, os usuários têm uma percepção global positiva, estando mais satisfeitos com o novo modelo de assistência na atenção primária. As falas dos entrevistados revelam um elevado grau de satisfação com o novo modelo assistencial marcado por sinais de melhoria do acompanhamento, maior segurança e com elevada partilha de informação e aposta em novas respostas. Tudo isso não acontecia no modelo anterior que estava condicionado por problemas de acesso, falta de comunicação entre os diferentes níveis de assistência e uma ausência de continuidade e não eram personalizadas nem direcionadas para a família.

Quando comparados os resultados do caso português com o Brasileiro (apresentado em estudo publicado no volume $14, \mathrm{n}^{\circ} 2$, da Revista da Universidade Vale do Rio Verde, Três

Corações) identificam-se pontos comuns como a melhoria na acessibilidade, no acolhimento e na resolubilidade, porém ambos os
- Maior acesso à informação clínica e programação da atividade em função das necessidades.

modelos poderão beneficiar adotando algumas práticas.

Como sugestão para pesquisas futuras, sugere-se a avaliação deste modelo em outros locais. Só por meio de estudos de avaliação da satisfação por meio da percepção dos usuários será possível melhorar a assistência em saúde e promover um melhor planejamento dos serviços melhorando as falhas e lacunas existentes.

\section{REFERÊNCIAS}

ACSS - ADMINISTRAÇÃO CENTRAL DO

SISTEMA DE SAÚDE. Relatório do acesso a cuidados de saúde nos estabelecimentos do SNS e entidades convencionadas. Lisboa: Ministério da Saúde.

ALMEIDA, Patty Fidelis de. FAUSTO, Márcia Cristina Rodrigues. GIOVANELLA, Lígia.

Fortalecimento da atenção primária à saúde: estratégia para potencializar a coordenação dos cuidados. Rev. Panam. Salud Publica. 2011; 29(2):84-95.

ASHCROFT, Rachelle. Health Promotion and Primary Health Care: Examining. Social Work in Public Health. 2015; 30(1):107-116.

ASSIS, Marluce Maria Araújo; JESUS, Washington Luiz Abreu de. Acesso aos serviços de saúde: abordagens, conceitos, políticas e modelo de análise. Ciênc Saúde Coletiva, Rio de Janeiro. 2012; 17(11): 2865-2875.

BAPTISTA, Makilim; CAMPOS, Dinael. Metodologias de Pesquisa em Ciências: Análises Quantitativa e Qualitativa. Rio de Janeiro: LTC, 2007.

BARDIN, Laurence. Análise de conteúdo. Lisboa: Edições 70, 2006. 
BISCAIA, André; HELENO, Liliana. Primary Health Care Reform in Portugal: Portuguese, modern and innovative. Ciência \& Saúde Coletiva. 2017; 22(3): 701-711.

BRASIL. Saúde da família: uma estratégia para a reorientação do modelo assistencial. Brasília: Ministério da Saúde, 1998.

CAMPOS, Rosana; FERRER, Ana; GAMA, Carlos; CAMPOS, Gastão; TRAPÉ, Thiago; DANTAS, Deivisson. Avaliação da qualidade do acesso na atenção primária de uma grande cidade brasileira na perspectiva dos usuários. Saúde Debate. 2014; 38 : 252-264.

CAR, Josip; SHEIKH, Aziz. Telephone consultations. BMJ. 2003; 326: 965-969.

CASTRO, Rodrigo Caprio Leite de; KNAUTH, Daniela Riva; HARZHEIM, Erno; DUNCAN, Bruce. Avaliação da qualidade da atenção primária pelos profissionais de saúde: comparação entre diferentes tipos de serviços. Cad. Saúde Pública. 2012, 28(9):1772-1784.

PORTUGAL. Decreto-Lei n. ${ }^{\circ}$ 298/2007, de 22 de agosto. Estabelece o regime jurídico da organização e do funcionamento das unidades de saúde familiar (USF). Diário da República n. ${ }^{\circ}$ 161/2007, Série I de 2007-08-22, p. 5587-5596.

FEIJÃO, Alexsandra; GIMENIZ, Galvão; MARLI, Teresinha. Ações de educação em saúde na atenção primária: revelando métodos, técnicas e bases teóricas. Revista da Rede de Enfermagem do Nordeste. 2007; 8(2): 41-49.

FERNANDES, Adalberto. A Combinação Público-Privado em Saúde: impacto no desempenho do sistema e nos resultados em saúde no contexto português (Tese de doutorado). Lisboa: Instituto Superior de Ciências Sociais e Políticas. Universidade de Lisboa, 2015.

FORTUNA, Cirina; OLIVEIRA, Karemme; FELICIANO, Adriana; SILVA, Mônica; BORGESM, Adriano; CAMACHO, Gabriela; ARAÚJO, Priscila; OGATA, Márcia. O acolhimento como analisador das relações entre os profissionais, gestores e usuários. Rev Esc Enferm USP. 2017; 51: 1-8.

GARUZI, Miriane. et al. Acolhimento na Estratégia Saúde da Família: revisão integrativa. Rev. Panam. Salud Pública 35(2), 2014.

KLEIN, John; GONÇALVES, Alda. A adesão terapêutica em contexto de cuidados de saúde primários. Psico-USF. 2005; 10(2): 113-120.
LIMA, Cássio; OLIVEIRA, Ana; MACEDO, Beatriz; DIAS, Orlene; COSTA, Simone. Relação profissionalusuário de saúde da família: perspectiva da bioética contratualista. Rev. bioét. 2014; 22 (1): 152-60.

MARTINS, Marisa; FONSECA, Franciele; MIRANDA, Leonardo; MENDES, Patrícia. Avaliação da atenção primária à saúde na perspectiva dos usuários diante da mudança do modelo assistencial. Revista da Universidade Vale do Rio Verde, Três Corações. 2016: 14(2): 1164-1174.

McMANUS, Alexandre. Health promotion innovation in primary health care. Australas Med J. 2013; 6(1): 15-18.

MENDES, Eugênio Vilaça. As redes de atenção à saúde. Brasília: Organização Pan-Americana de Saúde, 2011.

MESQUITA, Ana; CARVALHO, Emília. A Escuta Terapêutica como estratégia de intervenção em saúde: uma revisão integrativa. Rev Esc Enferm USP. 2014; 48(6):1127-36

MILAN, Gabriel Sperandio; TREZ, Guilherme. Pesquisa de satisfação: um modelo para planos de saúde. RAEletrônica. 2005; 4(2).

MINAYO, Maria Cecília. O desafio do conhecimento: pesquisa qualitativa em saúde. 13. ed., São Paulo: Hucitec, 2013.

NUNES, Alexandre. Reformas na Gestão Hospitalar: efeitos da empresarialização (Tese de doutorado). Lisboa: Instituto Superior de Ciências Sociais e Políticas. Universidade de Lisboa, 2016.

OMS - ORGANIZAÇÃO MUNDIAL DE SAÚDE. Aging and Health. World report on ageing and health. Copenhaga: OMS Publishing, 2015.

ONOCKO-CAMPOS, Rosana; CAMPOS, Gastão; FERRER, Ana; CORRÊA, Carlos; MADUREIRA, Paulo; GAMA, Carlos; DANTAS, Deivisson; NASCIMENTO, Roberta. Avaliação de estratégias inovadoras na organização da Atenção Primária à Saúde. Rev Saude Publica. 2012; 46(1): 43-50.

RAMOS, Donatela Dourado; LIMA, Maria Alice Dias da Silva. Health care access and receptivity to users in a unit in Porto Alegre. Cadernos de Saúde Pública. 2003; 19(1): 27-34.

SALES, Fátima. Ações de educação em saúde para prevenção e controle da dengue: um estudo em Icaraí, Caucaia, Ceará. Ciência \& Saúde Coletiva. 2008; 13(1):175-184.

SIMÕES, Jorge., AUGUSTO, Gonçalo., FRONTEIRA, Inês; Hernandez-Quevedo, Crisitna. 
(2017). Portugal Health System Review. Copenhagen: WHO Regional Office for Europe.

SIMÕES, Jorge; AUGUSTO, Gonçalo; FRONTEIRA, Inês. Introduction of freedom of choice for hospital outpatient care in Portugal: Implications and results of the 2016 reform. Health Policy. 2017; 121(12): 1203 1207.

SOUZA, Elizabethe; VILAR, Rosana; ROCHA, Nadja; UCHOA, Alice; ROCHA, Paulo. Acesso e acolhimento na atenção básica: uma análise da percepção dos usuários e profissionais de saúde. Cad. Saúde Pública. 2008; 24(Supl1): 100-110.

STARFIELD, Barbara. Atenção primária: equilíbrio entre necessidades de saúde, serviços e tecnologia. Brasília: Ministério da Saúde, 2002.

\footnotetext{
Alexandre Morais Nunes

Doutor em Administração da Saúde; Professor Auxiliar no Instituto Superior de Ciências Sociais e Políticas da Universidade de Lisboa; Investigador Integrado do CAPP; Assessor do Ministro da Saúde.
} 